\title{
Heart in An Eggshell Calcification: Idiopathic Calcific Constrictive Pericarditis
}

\author{
Bong Gun Songa, b Gu Hyun Kang ${ }^{\mathrm{a}}$, Yong Hwan Park ${ }^{\mathrm{a}}$, Woo Jung Chun ${ }^{\mathrm{a}}$, Ju Hyeon $\mathrm{Oh}^{\mathrm{a}}$
}

\begin{abstract}
Constrictive pericarditis is caused by fibrosis and calcification of the pericardium, which inhibits diastolic filling of the heart. Chest roentgenogram can show the calcification as a mass or sheet over the heart and computed tomography scan allows anatomic delineation of the pericardium and determines the extent of calcification. We reported a case of eggshell calcification of idiopathic chronic constrictive pericarditis diagnosed by echocardiography and multidetector computed tomography.
\end{abstract}

Keywords: Constrictive pericarditis; Echocardiography; Computed tomography

\section{Introduction}

Constrictive pericarditis (CP) is caused by fibrosis and calcification of the pericardium, which inhibits diastolic filling of the heart. Careful examination of chest roentgenogram may raise suspicion of calcific CP [1-4]. Computed tomography (CT) scan allows a nice anatomic delineation of the pericardium and determines the extent of calcification by comparing its morphology and density [5-6]. We reported an interesting

Manuscript accepted for publication November 17, 2011

aDivision of Cardiology, Cardiac and Vascular Center, Department of Medicine, Samsung Changwon Hospital, Sungkyunkwan University School of Medicine, Changwon, Korea

bCorresponding author: Bong Gun Song, Division of Cardiology, Cardiac and Vascular Center, Department of Medicine, Samsung Changwon Hospital, Sungkyunkwan University School of Medicine, Changwon, 630-522, Korea. Email: aerok111@hanmail.net

doi:10.4021/cr125w case of heavy eggshell calcification of idiopathic chronic CP diagnosed by echocardiography and multi-detector CT.

\section{Case Report}

A 41-year-old woman with no prior medical history visited our hospital for further evaluation of abnormal findings on two-dimensional transthoracic echocardiogram (TTE) performed during a routine health check-up examination. The patient showed mild dyspnea on ordinary physical activity with New York Heart Association class II and her physical examination was normal with a heart rate of 65 beats per minute and blood pressure of 110/60 mmHg. Initial electrocardiogram showed lower voltage complexes and chest roentgenogram revealed normal cardiac size with calcification of the pericardium (Fig. 1, arrows). On TTE, there were multiple spindle-like extensive calcifications in myocardium (Fig. 2, arrows). Left ventricle (LV) had normal chamber size (50 $\mathrm{mm}$ at end-diastole and $32 \mathrm{~mm}$ at end-systole) and wall dimensions (inter-ventricular septal wall thickness: 9 $\mathrm{mm}$ and LV posterior wall thickness: $9 \mathrm{~mm}$ ) and systolic function measured as $64 \%$. Mitral annular and aortic cuspal

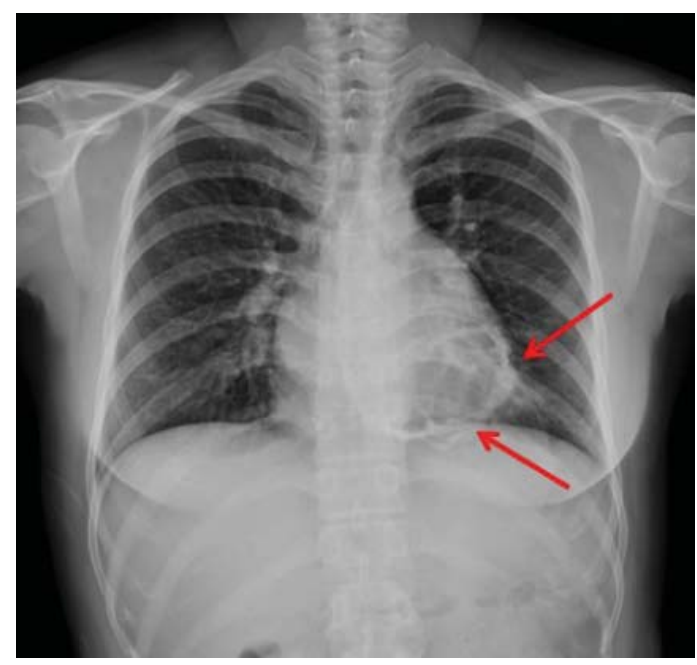

Figure 1. Chest roentgenogram showed normal cardiac size with calcification of the pericardium (arrows). 


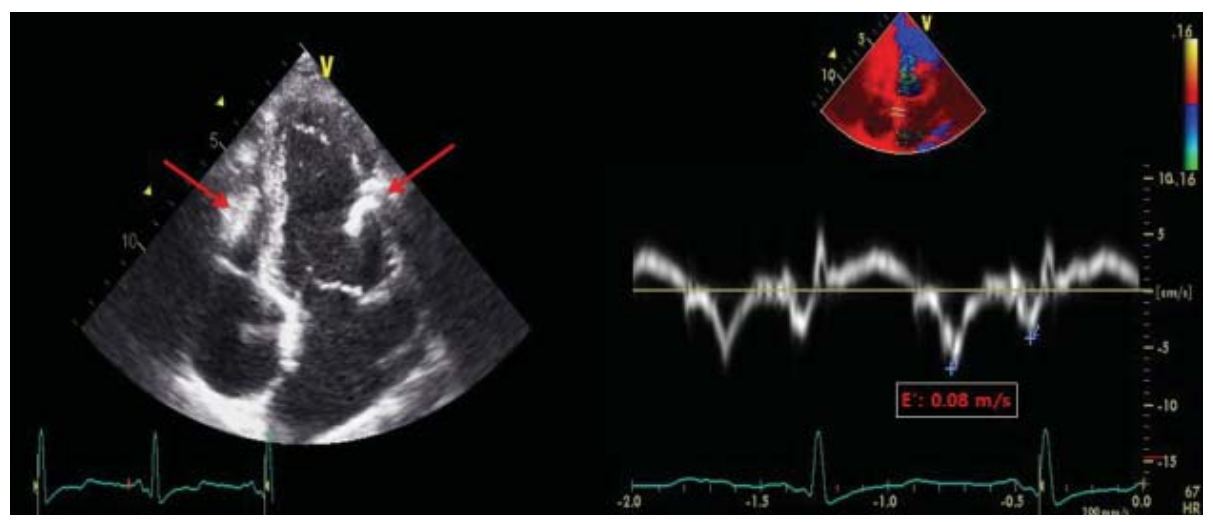

Figure 2. TTE showed multiple spindle-like extensive calcifications in myocardium (arrows). Tissue Doppler imaging revealed E'-velocity of the septal mitral annulus was $8 \mathrm{~cm} / \mathrm{s}$.

calcifications were not seen on TTE. Tissue Doppler imaging revealed that E'-velocity of the septal mitral annulus was 8 $\mathrm{cm} / \mathrm{s}$ indicating constrictive physiology (Fig. 2). TTE did not show significant valvular regurgitations or stenoses of more than mild grade. We performed 128-slice multi-detector CT, which demonstrated heavy eggshell calcification of the pericardium encircling the heart (Fig. 3). She refused open cardiac surgery and has been followed up with outpatient clinic visits.

\section{Discussion}

Pericardial calcification is thought to result from an inflammatory or traumatic event leads to fibro-calcific synechiae between pericardium and epicardium [1-4]. Up to two-thirds of all cases of pericardial calcification are unknown etiology [1-4]. Possible causative factors include Coxsackie B virus, radiation therapy, trauma, cardiac surgery, tuberculosis, malignancy, inflammatory and connective tissue diseases [1-4]. The presence of calcification denotes a chronic course where any causative factors induce a chronic intra-pericardial inflammation and subsequent healing with granulation tissue formation leading to development of adhesion and calcification. This in turn may cause the symptoms observed of CP [1-4]. Pericardial calcification is a common finding in patients with CP [1-3]. Careful examination of chest roentgenogram may raise the suspicion of calcific CP which showing the calcification as a mass or sheet over the heart $[1,7]$. Echocardiography is a relatively simple and highly sensitive technique to differentiate between CP and restrictive cardiomyopathy. An early diastolic velocity of the lateral or septal mitral annulus of $>8 \mathrm{~cm} / \mathrm{s}$ by pulse tissue Doppler is the generally accepted cut-off to differentiate $\mathrm{CP}$ and restrictive cardiomyopathy $[1,5]$. CT scan allows a nice anatomic delineation of the pericardium and its calcification [1, 5, 6]. Furthermore, CT best defines the asymmetric degree of pericardial thickening or calcification, which may be important in determining the optimal surgical approach for pericardial resection $[1,5,6]$. The standard treatment is surgery, which is usually achieved by pericardiectomy through a median sternotomy or lateral thoracotomy [1]. Our case has some interesting features. First, multiple spindle-like extensive calcifications in

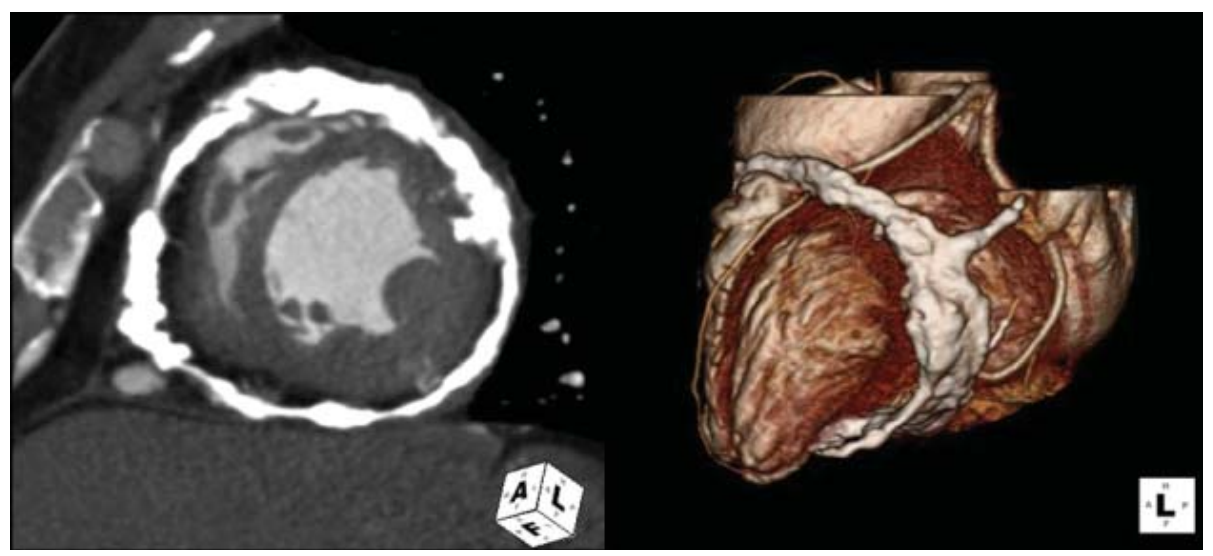

Figure 3. Multi-detector CT demonstrated heavy eggshell calcification encircling the heart. 
myocardium in this case is a rare finding in echocardiographic examinations. Second, pericardial calcification in our case is associated with idiopathic disease and is encircling right and left ventricles despite the pericardial calcification as described elsewhere was predominantly over the right atrium and ventricle, diaphragmatic surface, and AV grooves [1, 8]. We presented an interesting case of heavy eggshell calcification of idiopathic chronic CP diagnosed by echocardiography and multi-detector CT.

\section{Disclosure Statement}

The authors report no financial relationships or conflicts of interest regarding the content herein.

\section{References}

1. Ling LH, Oh JK, Breen JF, Schaff HV, Danielson GK, Mahoney DW, Seward JB, et al. Calcific constrictive pericarditis: is it still with us? Ann Intern Med. 2000;132(6):444-450.
2. Schiavone WA. The changing etiology of constrictive pericarditis in a large referral center. Am J Cardiol. 1986;58(3):373-375.

3. Cameron J, Oesterle SN, Baldwin JC, Hancock EW. The etiologic spectrum of constrictive pericarditis. Am Heart J. 1987;113(2 Pt 1):354-360.

4. Isaacs D, Stark P, Nichols C, Antevil J, Shabetai R. Posttraumatic pericardial calcification. J Thorac Imaging. 2003;18(4):250-253.

5. Sutton FJ, Whitley NO, Applefeld MM. The role of echocardiography and computed tomography in the evaluation of constrictive pericarditis. Am Heart J. 1985;109(2):350-355.

6. Reinmuller R, Gurgan M, Erdmann E, Kemkes BM, Kreutzer E, Weinhold C. CT and MR evaluation of pericardial constriction: a new diagnostic and therapeutic concept. J Thorac Imaging. 1993;8(2):108-121.

7. Steinberg I. Roentgenography of pericardial disease. Am J Cardiol. 1961;7:33-47.

8. Chambliss JR, Jaruszewski EJ, Brofman BL, Martin JF, Feil H. Chronic cardiac compression (chronic constrictive pericarditis); a critical study of sixty-one operated cases with follow-up. Circulation. 1951;4(6):816-835. 